\title{
Investigation of Split Flash Boiling Injection Schemes in a constant volume chamber and an optical GDI engine under lean combustion condition
}

\author{
Zhe Sun, Hongyu Wang, Mingli Cui, Mohamed Nour, Xuesong Li*, Min Xu* \\ School of Mechanical Engineering, Shanghai Jiao Tong University, Dongchuan Road 800 \\ Shanghai, China 200240 \\ ${ }^{*}$ Corresponding Authors: xuesonl@sjtu.edu.cn, mxu@sjtu.edu.cn
}

\begin{abstract}
Flash boiling atomization has the potential to enable superior spray atomization and more homogenous fuel-air mixing. These capabilities have been demonstrated in practical combustors such as gasoline direct-injection (GDI) engines. However, optimal injection schemes for flash boiling atomization have not been thoroughly investigated yet in the existing literature. In this work, we examined such contents in the aspects of split injection schemes coupled with flash boiling atomization. The impacts were examined firstly by velocity field analysis on single injection flash boiling plumes using particle imaging velocimetry (PIV). Furthermore, an optical reciprocating engine facility was used to study flame characteristics. Spray morphologies in the cylinder were studied, and the velocity field was visualized by the optical flow method. Important combustion characteristics, such as the flame speed and combustion duration, were investigated. Finally, the thermal efficiency under different injection schemes, as well as combustion emissions were quantified and compared to discuss the influence of flash boiling atomization on lean-operated GDI engines.
\end{abstract}

\section{Keywords}

Flash boiling atomization; Gasoline direct injection engines; Optical engine; Split injection

\section{Introduction}

The use of flash boiling fuel atomization is considered a viable path in further improving the combustion efficiency and reduce emission for practical combustors[1]. Such a capability is quite desirable under increasingly stringent regulations for applications such as gasoline direct injection (GDI) engine powertrains. To achieve flash boiling atomization, phase change needs to be enabled by reducing the local pressure of the fuel to a level lower than the local saturated vapor pressure. Usually, such conditions can be achieved by elevating the fuel temperature or by decreasing the ambient pressure. There have been many investigations on spray morphologies and characteristics in constant volume chamber tests, and the results have demonstrated that flash boiling atomization is able to improve fuel-air mixing[2], reducing droplet size [3], reducing spray-wall impingement fuel film formation [4, 5], etc. Despite the positive performance observed, there are also concerns with regards to the implementation of flash boiling spray, for instance, spray targeting change (spray collapse). The mechanisms for spray collapses are still not completely clear, and many explanations have been provided to illustrate the said physical process [6-9]. There are also investigations indicating that spray collapse may increase the amount of fuel impingement on the plate [10]. The seemingly conflicting results actually reflect that flash boiling is a quite complex physical phenomenon. Proper designs and optimization should be performed for flash boiling injection systems to reach their maximum potential in practical applications.

Flash boiling combustion has been practiced and investigated in IC engine settings, and an optical engine facility is commonly adopted in such investigations. Among these investigations, the capability of flash boiling sprays in addressing cold-start issues attracts notable attention in the field. Kale et al. [11] used three different types of fuels (iso-octane, n-butanol, and iso-butanol) under various fuel temperatures to study the flame characteristics in an optical engine. They found that Flash boiling can effectively atomize low volatile butanol isomers, and flash boiling can be helpful in reducing sooty combustions due to piston wall wetting. However, they also stated that spray collapse could increase the chances due to spray collapse. Kabasin et al. [12] used an integrated heated fuel injector for ethanol direct injection engines, and it was found that flash boiling effects notably improved spray breakup and cold-start performance. Similar observations were established by Huang et al. that combining port injection and direction injection schemes [13]. Fedor et al. [14] adopted a heated injector strategy combined with multiple injection schemes and found improvements in cold-start combustion characteristics. The work of Yang et al. explored flash boiling spray applications under engine cold-start conditions[2, 15, 16]. It was found that flash boiling combustion can significantly reduce soot emission, improve the indicative mean effective pressure (IMEP) of the engine, and improve cycle-to-cycle variation under cold-start conditions. 
Recently, besides cold-start related investigation, studies also focus on the impact of flash boiling sprays under warm conditions to demonstrate the ability of this technique in representative engine modes.

For instance, Mohamed et al. explored the impact of flash boiling atomization on butanol isomers with positive performance achieved [17, 18]. Comparable to flash boiling realizations under cold-start conditions, it was found that flash boiling atomization can reduce $\mathrm{CO}$, total hydrocarbon (THC), and particle emissions, meanwhile improve engine efficiency and cycle-to-cycle variation. Similar conclusions were found by Sun et al. [19, 20] when late injections or early injections were applied.

Although many investigations have been performed, the knowledge on flash boiling atomization is still immature. The breakup mechanisms and spray behaviors change tremendously so that new combustion systems/strategies should be studied to cater to the utilization of flash boiling atomization. Besides, new trends for IC engines also pose new challenges in adopting flash boiling atomization. For instance, the need for high thermal efficiency and compatibility with range extender systems require IC engines to operate under lean conditions so that the compression ratio can be further increased for GDI applications. Some preliminary investigations have been done by the authors in flash boiling atomization under lean-burn conditions[21, 22], but these studies are only demonstrative, and no thorough/systematic analysis has been performed. Therefore, in this work, we will focus on the injection strategy for flash boiling atomization under lean-burn conditions. More specifically, we will study the impact of split injection and split injection settings on spray characteristics and consequential combustion performance. The rest of the work is summarized as follows: Section 2 of this work introduces the experimental apparatus and methods for investigation, Section 3 presents the results and discussions are carried out, and finally, Section 4 concludes this investigation.

\section{Experimental Schematics}

The experimental facilities used in this work can be categorized into two sections. The first part of the experiments involves spray characterization in a constant volume chamber, with the experimental scheme depicted in Fig. 1. The goal of the experiments is to illustrate the flow field feature of the flash boiling spray so that the injection strategy can be designed accordingly. The PIV experiment provides the velocity distribution by taking the Miescattering signal from the seeding particles. Therefore, if the imaging plane has another liquid present simultaneously, the PIV data cannot distinguish them from the seeding particles. However, using the LIF method can effectively separate the two liquids in the spectral domain. Therefore, some researchers have combined the LIF with PIV to implement the FPIV experiment, sometimes called LIF-PIV [27].

Seeding particles Rhodamine 6G in polyethylene glycol (PEG 200) with the solution concentration of $1 \mathrm{~g} / \mathrm{L}$ were added into the measurement space using a 6-jet atomizer (TSI 9306). For the FPIV test, the most important consideration is to capture the particle's motion, so the particle's absolute intensity was not crucial. Therefore, the intensity correction was not applied in this experiment. The seeding particle density was $1270 \mathrm{~kg} / \mathrm{m} 3$ at $25^{\circ} \mathrm{C}$. The primary criteria of selecting the seeding particle were that the particle must be able to fully follow the air motion of the flow under the corresponding environment. Therefore, it requires that the particle size be as small as possible. However, if the drop size was too small, the particle may not scatter enough light. Therefore, the Stokes number and corresponding factors were calculated and shown in Table 1. Stokes number greater than 1 means the particle will detach from the flow when it suffers sudden change. Meanwhile, if the Stokes number was less than 1, the particle will wholly follow the fluid flow. The out-of-plane uncertainty was calculated in the $X$ (horizontal direction) and $\mathrm{Y}$-axis (vertical direction). The maximum perspective error in the $\mathrm{Y}$-axis direction was estimated to be $10 \%$, and the $\mathrm{X}$-axis was lower than $3 \%$. Therefore, the positions of the scattered light from seeding particles taken by the image pair correctly reflected the actual motion of the airflow. Thus, the displacement of the particles can accurately indications the flow motion.

As depicted in Fig.1, two cameras were used to capture the FPIV and Mie-scattering signal separately. An illumination laser sheet of $527 \mathrm{~nm} \mathrm{Nd:} \mathrm{YLF} \mathrm{(LDY303-PIV,} \mathrm{Litron)} \mathrm{was} \mathrm{formed} \mathrm{by} \mathrm{a} \mathrm{set} \mathrm{of} \mathrm{lenses} \mathrm{and} \mathrm{mirrors} \mathrm{were}$ directed into the test chamber. The laser pulse duration was $170 \mathrm{~ns}$, while the laser sheet width and thickness were $15.09 \mathrm{~mm}$ and $2 \mathrm{~mm}$. The Mie-scattering camera (Phantom V7.3, Vision) equipped with a Nikkor $50 \mathrm{~mm}$ lens (f/11) was used to capture the spray image. The laser energy was about $2 \mathrm{~mJ} /$ pulse at each laser head, so the Miescattering signal was powerful. That is the reason why a small aperture is used for the spray camera.

Another high-speed camera (Phantom V1210, Vision) was used to capture the LIF signal by putting a $550 \mathrm{~nm}$ longpass filter in front of the camera lens. The lens is a Nikkor $105 \mathrm{~mm}$ of $\mathrm{f} / 2.8$. A dichroic mirror that reflects $99 \%$ light lower than $550 \mathrm{~nm}$ was used to adjust the light path and enhances the wavelength separation to reduce the crosstalk. The two cameras and the fuel injector were synchronized by a high-speed controller (HSC, LaVision) connected with a computer. For this study, we are primarily interested in the air entrainment process induced by the flash boiling spray (since it alters the structured flows inside the engine).

The spatial scales of the two cameras have been calibrated by putting a calibration plate at the laser plane. The Mie-scattering camera has a field-of-view of $30.41 \times 28.51 \mathrm{~mm} 2(640$ pixels $\times 600$ pixels $)$ at a spatial resolution of 
$0.048 \mathrm{~mm} /$ pixel. The PLIF camera has a field-of-view of $15.09 \times 13.46 \mathrm{~mm} 2$ (896 pixels $\times 800$ pixels) at a spatial resolution of $0.017 \mathrm{~mm} /$ pixel. The images from the two cameras were overlapped, and therefore a direct comparison of the Mie-scattering and FPIV images can be achieved.

Thirty cycles of high-speed imaging sequence have been recorded at $5 \mathrm{kHz}$ for each test condition. The FPIV camera captured the double-frame image pair, while the Mie-scattering camera captured a single spray image, as shown in the right part of Fig.1. The fuel injection duration is $1.0 \mathrm{~ms}$, and the spray almost vanishes after $1.5 \mathrm{~ms}$ aSOF.

A specially designed water jacket in the chamber testing was used to heat the injector body. The temperatures of water and injector inlet were measured. Furthermore, an injector with a thermocouple installed inside the injector body was utilized to calibrate the controlling temperature and precisely monitor the fuel temperature before injection at the desired value. Other experimental setup can be found in our previous study [27-29].

The single-hole GDI injector (Delphi) was used in the chamber testing to avoid the influence of plume-to-plume interaction and focus on the influence flash boiling phenomenon on the spray. The ambient pressure is $40 \mathrm{kPa}$, and the temperature is $25^{\circ} \mathrm{C}$. We made a dimensionless number $\mathrm{Pa} / \mathrm{Ps}$ to describe the degree of superheat in the previous studies (a summary could be found in reference [30]), and the demarcation point was found to divide the spray condition into three conditions: subcooled spray, transitional flash boiling spray, and flare flash boiling spray. In transitional flash boiling spray, features like droplets size and the configuration will be susceptible to the fuel temperature. However, once reach the temperature to make the spray translated to flare flash boiling spray, there is no significant difference with the upper temperatures. Therefore, in the chamber testing, $90^{\circ} \mathrm{C}$ was under flare flash boiling, and $30^{\circ} \mathrm{C}$ was under subcooled spray condition corresponding to the ambient pressure so that the comparison was obvious. The seeding particle properties, ambient condition, and injection parameters have been summarized in Table 1.

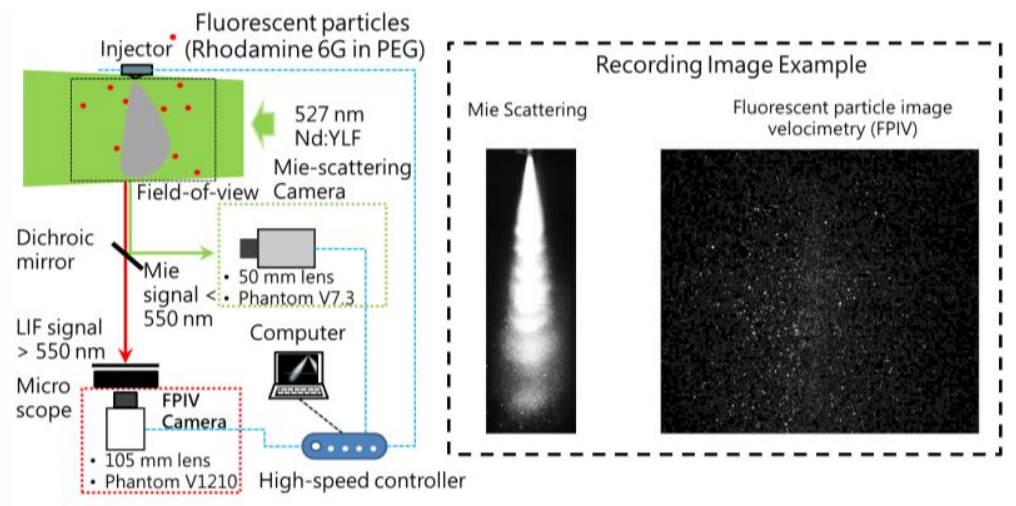

Figure 1. Experimental setup for flash boiling spray air entrainment studies and its recording example.

Table 1 Chamber specifications and experimental conditions

\begin{tabular}{ll}
\hline Category(unit) & Value \\
\hline Seeding Particle Properties & \\
Particle density $\left(\mathrm{kg} / \mathrm{m}^{3}\right)$ & $1270 @ 25^{\circ} \mathrm{C}$ \\
Particle diameter $(\mathrm{m})$ & $\sim 5 \times 10-6$ \\
Air dynamic viscosity $(\mathrm{Pa}-\mathrm{s})$ & $18.5 \times 10-6 @ 25^{\circ} \mathrm{C}$ \\
Characteristic velocity $(\mathrm{m} / \mathrm{s})$ & 20 \\
Characteristic length $(\mathrm{m})$ & 0.01 \\
Stokes number & $1.91 \times 10-1$ \\
\hline Ambient Condition & \\
Pressure $(\mathrm{kPa})$ & 40 \\
Temperature $\left({ }^{\circ} \mathrm{C}\right)$ & 25 \\
\hline Injector\&Injection Parameters & 1 -hole $(($ Delphi) \\
Injector Type & Conmercial Gasoline $(92)$ \\
Fuel Type $(\mathrm{RON})$ & 5 \\
Pressure $(\mathrm{MPa})$ & 1 \\
Duration $(\mathrm{ms})$ & $30-90$ \\
Temperature $\left({ }^{\circ} \mathrm{C}\right)$ & \\
\hline
\end{tabular}


For the combustion tests, an optical engine facility was used, and the systematic of the optical engine system is illustrated in Fig 2. As shown, in this work, we used a heating element wrapping the fuel rail to heat up the fuel and generate flash boiling sprays. The fuel temperature was measured and monitored by a thermocouple with a PID control module. The optical engine used was a single-cylinder one. Basically, the compression ratio of the engine was a moderate one (10.5), and the engine was operated at low-speed conditions. The combustion performance under lean conditions was examined, and thus high-energy ignition method was used to assist the combustion process. An extended ignition current scheme was used (see [22, 23] for detailed information for this constant current scheme) with an ignition duration of $2 \mathrm{~ms}$ and ignition energy of $826 \mathrm{~mJ}$. The piston and the cylinder liner can switch between quartz ones and metal ones for different diagnostics purposes. For instance, a quartz optical piston can be used together with a metal liner so that optical measurements can be performed through the Bowditch mirror beneath the optical piston. The rest of the engine conditions will not be explicated, and they have been summarized in Table 1.

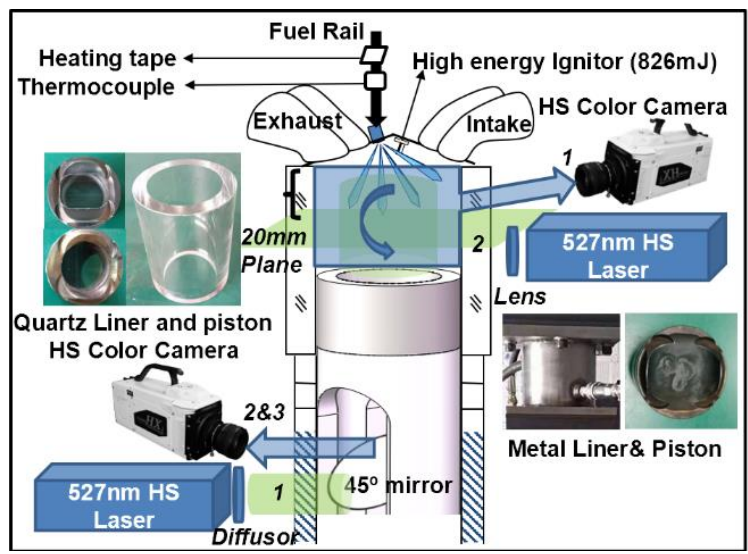

Figure 2. Optical arrangements for the optical engine measurements and definitions for the combustion images obtained from high-speed cameras.

Table 1 Engine specifications and experimental conditions

\begin{tabular}{|c|c|}
\hline Parameter (unit) & Value \\
\hline Displacement $\left(\mathrm{cm}^{3}\right)$ & 400.17 \\
\hline Bore $\times$ stroke $(\mathrm{mm})$ & $75.1 \times 90.34$ \\
\hline Compression ratio & 10.5:1 \\
\hline Speed $(\mathrm{rpm})$ & $800 / 1500$ \\
\hline Lubricant/coolant temperature $\left({ }^{\circ} \mathrm{C}\right)$ & $60 \pm 1$ \\
\hline Fuel type & Gasoline (RON 92) \\
\hline Injector type & GDI Bosch, 8 holes \\
\hline Intake timing (IVO/IVC) ('TTDC) & $357 / 74$ \\
\hline Exhaust timing (EVO/EVC) ( $\left.{ }^{\circ} \mathrm{bTDC}\right)$ & $86 / 390$ \\
\hline Fuel injection pressure (MPa) & 10 \\
\hline Spark timing $\left({ }^{\circ} \mathrm{bTDC}\right)$ & 56 \\
\hline Discharge parameters & $2 \mathrm{~ms}, 826 \mathrm{~mJ}$ \\
\hline Injection timing $\left({ }^{\circ} \mathrm{bTDC}\right)$ & $\begin{array}{c}\left.300 \text { (single and split } 1^{\text {st }}\right) \\
\left.\text { Various (Split } 2^{\text {nd }}\right)\end{array}$ \\
\hline Injection duration $(\mu \mathrm{s})$ & 2500 (single) /1250\&1250 (split) \\
\hline Intake manifold absolute pressure $(\mathrm{kPa})$ & 90 \\
\hline Lambda & 1.55 (single) and 1.57(split) \\
\hline Tumble Ratio & 3.2 \\
\hline
\end{tabular}

\section{Results and Discussion}

\section{Air Entrainment by Single Plume Flash Boiling Spray}

As discussed, to maintain the consistency of spray testings and engine testings, the same type of fuel (gasoline, RON 92) was used. For multi-component fuels, it is challenging to well define the exact boiling temperature. According to [15], we used a temperature of $90^{\circ} \mathrm{C}$ as the flash boiling temperature. It is also worth noting that the light components would vaporize even at lower temperatures; thus, the impact of the fuel temperature on spray-air 
entrainment needs to be investigated. Figure 3 exhibits the velocity field of the ambient air under subcooled spray injection conditions $\left(30^{\circ} \mathrm{C}\right)$ and flash boiling spray injection conditions $\left(90^{\circ} \mathrm{C}\right)$, respectively. The time duration before $1.0 \mathrm{~ms}$ aSOI (after start of injection) could be considered as the injection period. The time afterward may be considered as the after injection period since the spray had left the observation zone of PIV measurements. The morphologies of the spray plumes measured simultaneously were used as the background to demonstrate the location of the sprays.

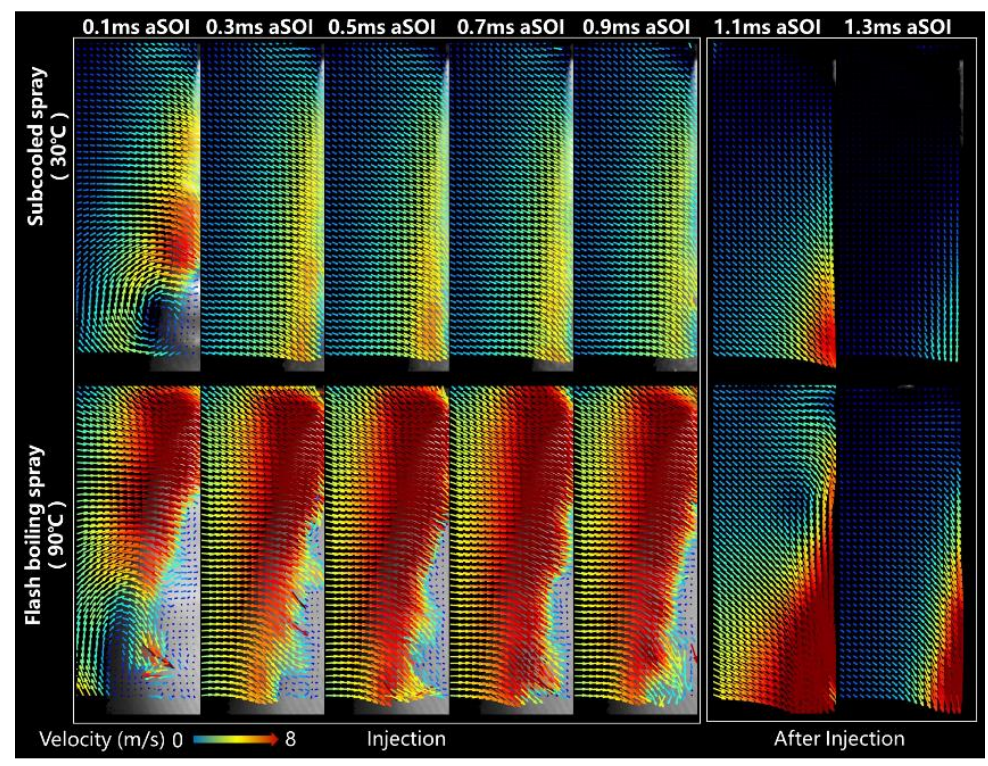

Figure 3. Ambient air velocity distribution under different spray thermodynamic conditions.

As seen in Fig. 3, the air velocity distribution of flash boiling spray under flash boiling conditions is distinctive from that under sub-cooled conditions. Under subcooled conditions, the spray plume induced vortices at the tip of the plume (as seen at $0.1 \mathrm{~ms}$ aSOI). While as the spray tip moved out of the measurement zone, the ambient air motion was uniform and followed the motion of the spray plume. It can be seen that the dopant droplets penetrated into the spray plume under these conditions. In comparison, for flash boiling spray injections, the plume width was larger, and a greater vortex was formed at $0.1 \mathrm{~ms}$ aSOI. Furthermore, the ambient air could not completely penetrate the flash boiling spray plume in later injection phases. The airflow field was also notably less uniform than that under subcooled conditions, indicating a stronger interference impact of the flash boiling spray to the ambient air. To quantify the impact of spray plume on the ambient air, we calculated the average velocity variation with various fuel temperatures, and the results are incorporated in Fig. 4. Since the velocity measurements of dopant droplets entrained into the spray plume might directly follow the spray motion, in this analysis, we have removed the velocity measurements inside the spray plume (as indicated in Panel (a) of Fig. 4), then calculates the average of the rest of the velocity magnitudes, reflected by the green arrows in Panel (a) of Fig. 4. The averaged velocities as a function of time are shown in Panel (b) of Fig. 4. A fuel temperature increment of $5^{\circ} \mathrm{C}$ from $30{ }^{\circ} \mathrm{C}$ to $90{ }^{\circ} \mathrm{C}$ was used to examine the influence of fuel temperature on air entrainment. At early injection times $(<0.5 \mathrm{~ms}$ aSOI), the entrainment efficiency of subcooled spray is slightly more pronounced than flash boiling sprays. During late injection times ( 0.5-0.9 ms aSOI), the air velocity grew slightly higher under flash boiling conditions than subcooled conditions. As the spray propagated past the imaging zone (>1.1 ms aSOI), wakes with notably higher velocities can be observed under flash boiling conditions. As can be seen from Panel (b) of Fig. 4 , the averaged velocity increased monotonously as a function of fuel temperature, and the velocity magnitude doubled (from $\sim 0.75$ $\mathrm{m} / \mathrm{s}$ to $1.50 \mathrm{~m} / \mathrm{s}$ ) as the fuel temperature increased from $30^{\circ} \mathrm{C}$ to $90^{\circ} \mathrm{C}$. Such observation indicates that flash boiling would produce a stronger wake, which could further promote fuel-air mixing in the cylinder. 

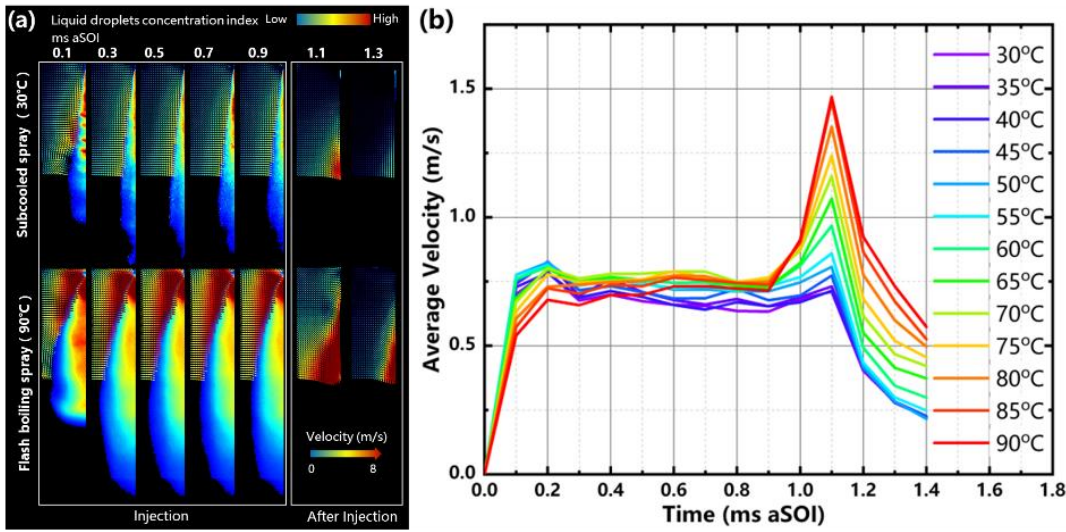

Figure 4. Ambient air velocity variations from the impacts of spray injection.

To understand the entrainment details under flash boiling conditions, we examined the flash boiling spray plume with a fuel temperature of $90^{\circ} \mathrm{C}$ at $0.1 \mathrm{~ms}$ aSOI, as shown in Panel (a) of Fig. 5. We are particularly interested in the primary plume zone (as indicated by the upper box in Fig. 5) and the plume tip zone (as reflected by the lower box). As can be seen, in the primary plume zone, the air penetrated deeply into the spray, while the penetration could not completely offset the expanding plume width, as can be identified in the lower box. Furthermore, stronger vortex motions were seen in the lower box. To better quantify and illustrate the impact of flash boiling entrainments, Panel (b) of Fig. 5 depicts the radial/horizontal velocity distributions under subcooled and flash boiling conditions at various timings. The same color scale was used for all plots. As can be clearly noted, the radial velocity under flash boiling injection conditions was substantially greater than that under subcooled conditions. In addition, strong entrainment took place closer to the injector tip under flash boiling conditions, while the entrainment of subcooled sprays located downstream of the spray plume. Finally, the flash boiling spray created strong entrainment even in late injection timings (1.1 ms aSOI). Such observations further attest to the conclusion that flash boiling atomization can notably improve fuel-air mixing and enhance turbulence in the cylinder.

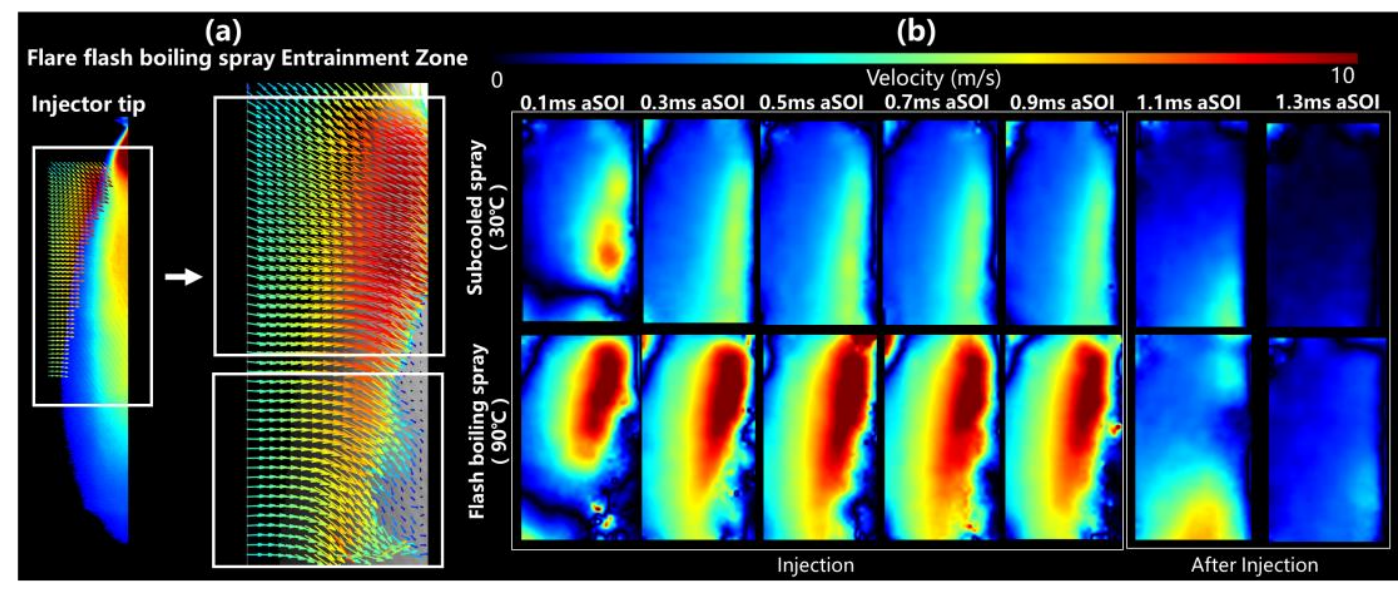

Figure 5. Panel (a). Strong ambient air entrainments induced by flash boiling spray injections. Panel (b). Radial/horizontal velocity magnitude distributions induced by spray entrainment.

\section{Spray Characteristics in Optical Engine}

Figure 6 depicts representative spray morphologies in the optical cylinder measured through the quartz liner installed. Note that the results are just for illustrative purposes; as such, no distortion correction process is done to address the refractive effect of the optical liner. In Fig. 6, macroscopic spray patterns are shown under both subcooled injections and flash boiling injections. The sprays created by various injection strategies were also shown and compared. Four representative timing, including 1.8 CA aSOI, 4.5 CA aSOI, 7.2 CA aSOI, and 9.9 CA aSOI, were used to depict the spray developments under each condition. For split injection cases, the spray morphologies for the second injection were also shown in the same manner. As can be seen, for single injection conditions or the first injection from the split injection schemes, the spray morphologies under sub-cooled conditions and flash boiling conditions were similar, and they all collapsed into one single plume. Discussions on the difference in spray patterns will be incorporated in the sections that follow. As for the second injection, remarkable differences can be seen in the results. Firstly, for the injection timing of $280^{\circ} \mathrm{bTDC}$ and 240 oTDC, the sprays still collapsed under both subcooled conditions and flash boiling conditions. For injection timing of $180^{\circ} \mathrm{bTDC}$ and $120^{\circ} \mathrm{bTDC}$, 
the spray plumes were recognizable under both subcooled conditions and flash boiling conditions, with no apparent spray collapse took place in early injection times. Noting that the ambient pressure was low ( $90 \mathrm{kPa})$ before $120^{\circ} \mathrm{bTDC}$ and then elevated after $120^{\circ} \mathrm{bTDC}$, it can be seen that the ambient pressure is a critical parameter in determining whether the spray is collapse, no matter under subcooled conditions or flash boiling conditions. Furthermore, significant vortex motion can be seen at the spray tips under flash boiling conditions in comparison to those under subcooled conditions, which we would elaborate on in greater detail as follows.

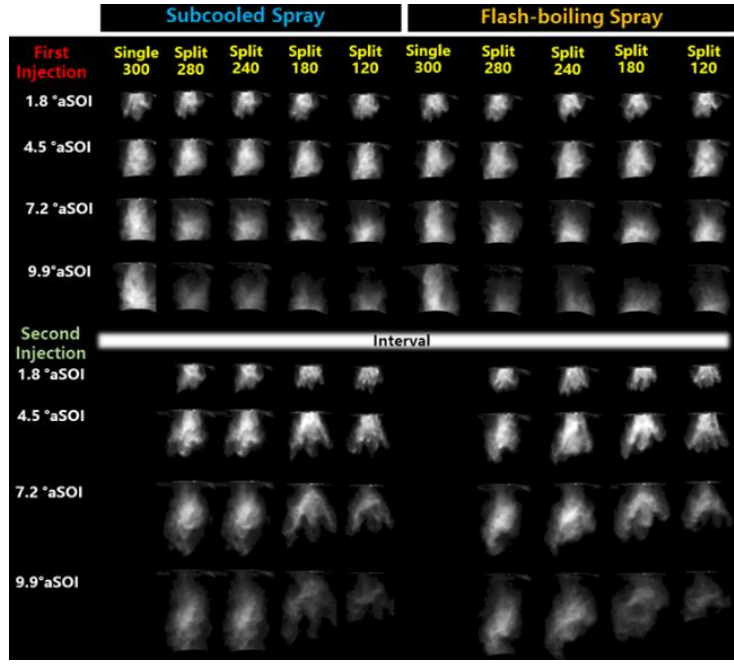

Figure 6. Spray morphologies inside the optical engine using different injection schemes.

To interpret in-cylinder spray atomization performance statistically, we performed a spray presence probability index (PPI) analysis for both single injection scheme and split injection scheme, and corresponding results are incorporated in Fig. 7. The PPI in each pixel of the image represents the likelihood that the spray occurs in this pixel at a specific time. 100 fuel injection cycles were used for the analysis. The injection timing for single injection cases was 300 'bTDC; the injection timing for the split injection case was $300^{\circ} \mathrm{bTDC}+280^{\circ} \mathrm{bTDC}$. The times selected for PPI comparison were $285.6^{\circ} \mathrm{bTDC}$ and $275.7^{\circ} \mathrm{bTDC}$ (split injection cases). As can be seen, for the three scenarios shown in Fig. 7, the PPI values under flash boiling spray atomization were higher. We calculated the $>90 \%$ area ratio (the number of pixels with a PPI $>0.9$ divided by the total number of pixels where spray occurred) and found that flash boiling atomization produced the $90 \%$ area ratio around $0.8 \%-2.0 \%$ higher than subcooled atomization cases, indicating the flash boiling spray is able to generate more stable vaporization processes.

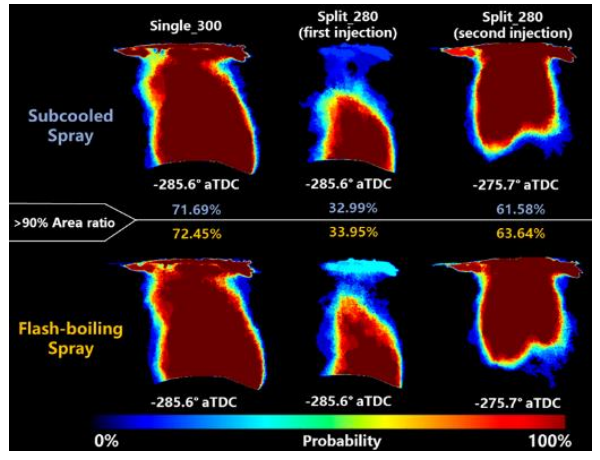

Figure 7. Spray presence probability index (PPI) distribution under different injection conditions.

Figure 8 and Figure 9 depict the velocity fields inside the optical engine during engine compression under single injection and split injection schemes, respectively. PIV velocity measurements of multi-hole sprays are challenging due to the complication imposed by the optical cylinder. Therefore, in this study, we used the optical flow method $[24,25]$ to evaluate the behaviors of the spray. Representative image shots were selected to demonstrate the difference between subcooled atomization and flash boiling atomization. It is worth noting that the observations are generally valid among all measurements. As reflected in Fig. 8, distinctive spray structures and flow fields are seen under different thermodynamic states of the fuel. In early injection phases (290.43 $\left.{ }^{\circ} \mathrm{bTDC}\right)$, under subcooled conditions, the flow field of the spray exhibits a downward trend, which would lead to direct impact onto the engine piston. Under flash boiling conditions, as highlighted by the dash circles, the spray propagated more significantly in the radial direction. In the circle on the left, it can be spotted that stronger entrainment took place under the 
impact of the tumble flow. The tumble flow also drove the spray towards the engine wall, as denoted in the dashed circle on the right-hand-side. Consecutively (at the timing of 288.69 'bTDC), different spray morphologies can be identified under subcooled and flash boiling conditions. The spray under flash boiling conditions was notably distorted under flash boiling conditions in an "S" shape. We consider such observations the results of structured tumble flows inside the engine and a lower Stokes number of the fuel droplets. Subsequently, the vortex structure is more pronounced under flash boiling conditions, which is desirable for fuel-air mixing and reducing spray impingement onto the piston head.

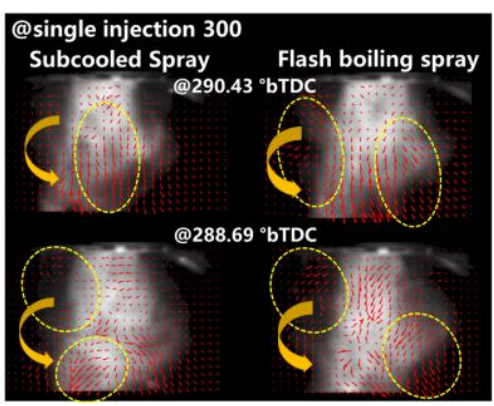

Figure 8. Optical flow quantifications of spray flow fields inside the optical engine facility (single injection). Yellow arrows represent the tumble motion inside the engine cylinder.

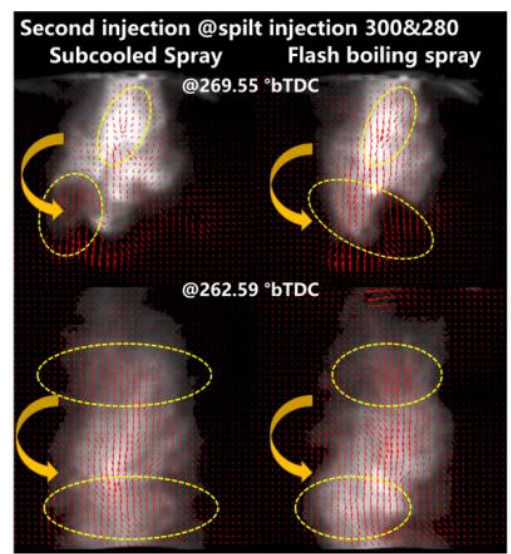

Figure 9. Optical flow quantifications of spray flow fields inside the optical engine facility (split injection, second injection). Yellow arrows represent the tumble motion inside the engine cylinder.

Figure 9 compares the spray morphologies and velocity fields given by the optical flow method under subcooled conditions and flash boiling conditions, respectively. The results for the first injection out of the two injections were not shown since the observations were similar to those in the single injection cases. Note that the injection timing of the second injection was $280.0^{\circ} \mathrm{bTDC}$. At the engine timing of $269.55^{\circ} \mathrm{bTDC}$, the spray plumes under subcooled conditions slightly collapsed, but the plumes were generally distinctive. The velocity vectors of the spray were primarily directed downward, indicating a negligible impact from the surrounding flows. In contrast, the flash boiling spray plumes were significantly distorted at the tip, suggesting more apparent impacts from the tumble flow or from the wake of the first injection. Similar observations are seen in the spray images at $262.59{ }^{\circ} \mathrm{bTDC}$. The radial velocity magnitudes in flash boiling atomization cases were greater than those in the subcooled conditions. Furthermore, under flash boiling conditions, the spray density was low at the tip of the spray plumes (especially on the right-hand-side of the spray). Such a feature is helpful in reducing spray-wall impingement even in split injection cases.

To briefly summarize, the structure of flash boiling sprays inside the optical engine is easier to be impacted by the structured flows inside the cylinder. Flash boiling atomization also enhances fuel-air mixing and reduce cycle-tocycle variations of the engine being studied. In the next section, we will continue studying the combustion performance with the characteristics of spray atomization in mind.

\section{Combustion Performance using Flash Boiling Atomization}

Representative flame development processes under different injection schemes are summarized in Fig. 10. The spark timing was $56.0^{\circ} \mathrm{bTDC}$, and we present the flame developments from $21.5^{\circ} \mathrm{bTDC}$ until the flame completely filled the optical window in the piston. The flame development under both subcooled conditions and flash boiling conditions are plotted. Single-injection (300 $\left.{ }^{\circ} \mathrm{bTDC}\right)$ and split injection (300 $\left.{ }^{\circ} \mathrm{bTDC}+280^{\circ} \mathrm{bTDC}\right)$ schemes were also examined. It is worth noting that we chose this split injection scheme for subcooled conditions to compare with 
flash boiling combustion performance, while this scheme might not be optimal for sub-cooled conditions. As can be seen, the flame speed using flash boiling atomization was faster in both single injection and split injection cases, which is in agreement with our previous work[21]. Furthermore, in subcooled injection conditions, hot spots can be spotted from the optical window at $17.5^{\circ} \mathrm{aTDC}$, indicating the existence of incomplete combustion or inadequate fuel-air mixing under these conditions. The flash boiling flame speed using the split injection scheme was slightly faster than single injection cases. Color-wise, more red flame components were seen under flash boiling conditions, which is in agreement with our color-based combustion analysis[20]. Finally, there are also yellow emissions observed in split injection cases near the spark plug. We hold that this observation is caused by the metal reflection of the spark plug in early engine timings, i.e., before $0^{\circ}$ aTDC.

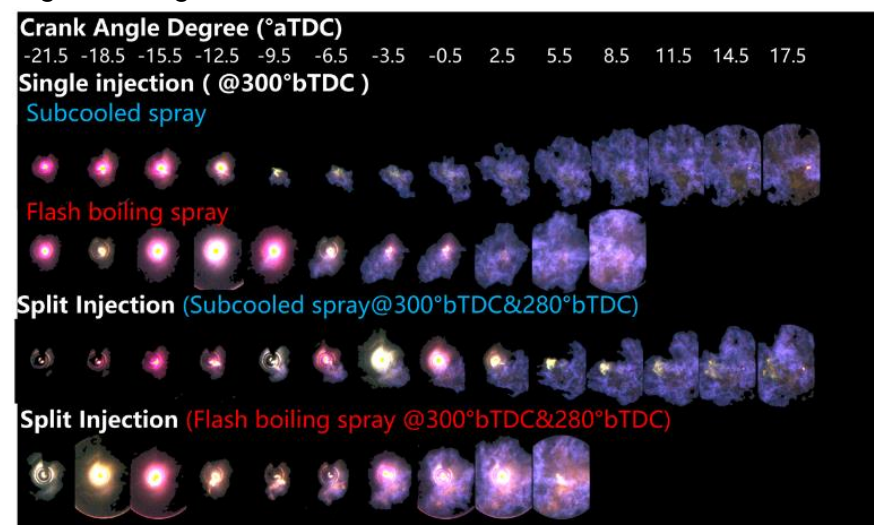

Figure 10. Cross-sectional flame propagation using various injection schemes.

Figure 11 demonstrates the combustion characteristics in the aspect of cylinder pressure curves and combustion durations. Panel (a) of Fig. 11 shows the pressure curves as a function of engine crank angles under various conditions. As can be seen, the onset of combustion under all three conditions was similar, while single injection, flash boiling atomization improved the maximal pressure notably, indicating that flash boiling atomization can contribute to better combustions under lean conditions. Furthermore, split injections can further enhance the maximal pressure, as seen from the results. It is worth noting that the enhancement is more significant under lean combustion conditions when the homogeneity of the fuel mixture is critical. Such improvements are not quite remarkable under stoichiometric conditions[22]. Panel (b)- Panel (d) denotes the combustion durations under different injection strategies, in which CA10 is defined by the crank angles from ignition to $10 \%$ burn, CA50 from ignition to $50 \%$ burn, and CA 1090 from $10 \%$ to $90 \%$ burn. As we can see, flash boiling atomization achieved shorter combustion periods as the second injection was earlier than $210{ }^{\circ} \mathrm{bTDC}$, while the combustion durations under flash boiling conditions were at the same level as sub-cooled conditions as the second injection was later than $210^{\circ} \mathrm{bTDC}$. Furthermore, the combustion duration reached the maximum at $210{ }^{\circ} \mathrm{bTDC}$, which implies a worse combustion performance for gasoline engines. The probable causes and consequences of this observation will be discussed below.
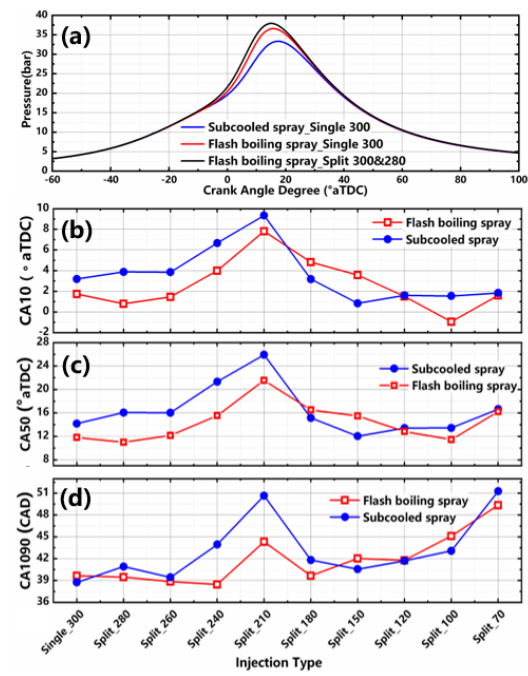

Figure 11. Combustion characteristics under various injection schemes. Panel (a). In-cylinder pressure variation as a function of crank angle, Panel (b). CA10 as a function of injection duration, Panel (c). CA50 as a function of injection duration, Panel (d). CA10-90 as a function of injection duration. 
Figure 12 demonstrates the indicated mean effective pressure (IMEP) and the coefficient of variation (COV) of IMEP, respectively. Both flash boiling and subcooled injections were considered. As we can see, the IMEP using flash boiling atomization is notably higher than that under subcooled conditions. For subcooled conditions, the IMEP was generally lower using split injection schemes than single injection schemes, which is somehow reasonable since the main purpose of using split injection is to reduce wall impingement. For flash boiling conditions, peak IMEP was found using the split injection scheme with the injections at $300{ }^{\circ} \mathrm{bTDC}$ and $280{ }^{\circ} \mathrm{bTDC}$. We hold that such improvements are attributed to stronger air entrainment motions and remained vortex strength at the time of the second/adjacent fuel injections. We found that the worst IMEP also occurred at $210{ }^{\circ} \mathrm{bTDC}$, which is in agreement with the results shown in Fig. 11. It is believed that this phenomenon is caused by the weak structured flows near $180^{\circ} \mathrm{bTDC}$, as the piston moves near the bottom dead center. As the spray propagates since $210^{\circ} \mathrm{bTDC}$, the fuel-air mixing and evaporation deteriorate, thus extend the combustion duration and reduce the IMEP of the engine. The same rationale can be adopted to analyze IMEP COV. When the second injection was earlier than $210^{\circ} \mathrm{bTDC}$, flash boiling assists to reduce cycle-to-cycle variations compared with subcooled atomization. However, as the second injection is late, the time left for spray evaporation and combustion is limited. Thus the IMEP COV is considerably increased under both subcooled and flash boiling conditions. At this period, backpressure was higher, and the flash boiling phenomenon was suppressed. Both conditions had similar spray features. The airflow was reconstructed, and the tumble flow was strengthened again. There was a trade-off between the in-cylinder distribution and the remaining evaporation time of the fuel. Complicated air-fuel interaction combined with the remaining evaporation time during the compression injection resulted in higher combustion variations, even including the air-wall interaction.

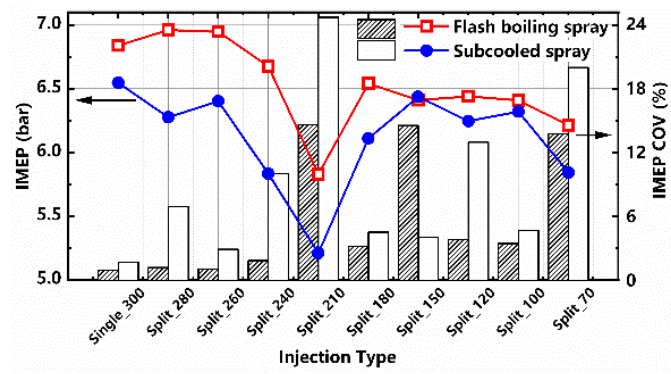

Figure 12. IMEP performance and cycle-to-cycle variation under different conditions.

Figure 13 shows the normalized thermal efficiency under different injection strategies. Although the thermal efficiency of optical engines can usually be lower than practical internal combustion engines due to leaking or design issues, it is safe to compare the efficiency with a preset benchmark. In this work, we set the thermal efficiency with subcooled fuel single injection as the benchmark (100\%) and scaled those under the other conditions. As seen, the normalized thermal efficiency was improved with the second injection at $260{ }^{\circ} \mathrm{bTDC}$ and $150{ }^{\circ} \mathrm{bTDC}$, but the increments were subtle. The normalized thermal efficiency under flash boiling conditions are all higher compared with the benchmark except for the case with the second injection at 240 obTDC, and thermal efficiency improvement with the second injection before 210 obTDC was notably higher than the benchmark, indicating the advantage of adopting two adjacent injections under flash boiling conditions.

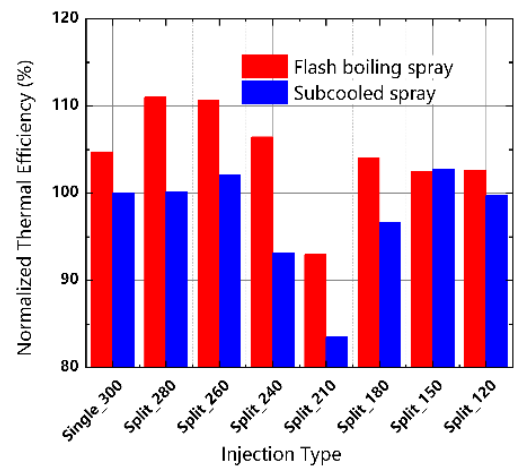

Figure 13. Normalized thermal efficiency using different injection schemes.

Finally, we examined the emission characteristics under flash boiling combustion conditions, and the results are incorporated in Fig. 14. Before any discussion, it should be noted that the combustion was under lean conditions. Thus the emissions were relatively low and more acceptable generally compared with stoichiometric cases. In this investigation, total hydrocarbon (THC) emissions, CO emissions, NOx emissions, and particle number (PN) emissions are listed and compared in Panel (a)- Panel (d) of Fig. 14, respectively. As seen, the THC, CO, NOx 
emissions under flash boiling combustions were mostly lower than sub-cooled conditions, except for the THC emission with the second injection at $210{ }^{\circ} \mathrm{bTDC}$. For PN emissions, flash boiling cases are generally better with the second injection before $210^{\circ} \mathrm{bTDC}$, but worse with the second injection after $210^{\circ} \mathrm{bTDC}$. These results are intuitive except for the NOx emission, as the flash boiling atomization will increase the combustion temperature as a whole. Our explanation is that under lean combustion cases, the overall temperature is lower than that in stoichiometric cases. Therefore, a moderate rise in overall temperature would have negligible influences on NOx emission. In contrast, local inhomogeneity and hot spots can be regarded as the primary sources of NOx emission under lean cases, while flash boiling sprays can suppress these negative effects. Therefore, the NOx emission performance improves instead under flash boiling conditions with a lean combustible mixture.
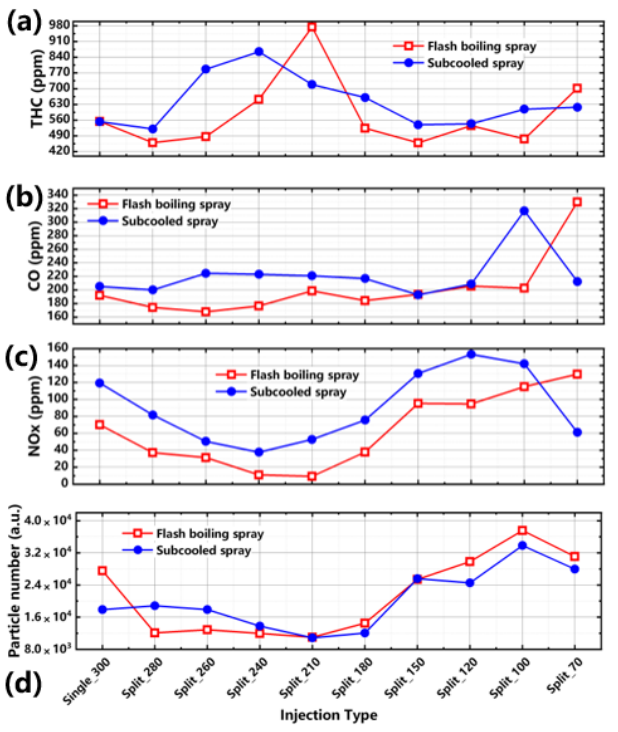

Figure 14. Emission behaviors under different conditions and injection schemes. Panel (a). Total hydrocarbon (THC), Panel (b). CO, Panel (c). NOx, Panel (d), Particle number (PN).

\section{Conclusion}

In this work, we investigated split injection schemes under flash boiling conditions, and positive effects are established. The conclusions are listed as follows:

1. The air entrainment of flash boiling spray plume is much stronger compared with subcooled spray plume. Notable vortex structures form both at the plume tip and the wake after spray propagations.

2. Combustion characteristics such as the flame propagation speed and combustion duration are improved with flash boiling atomization. Split injection with a short gap between two adjacent injections achieved an even superior combustion performance.

3. The emission properties under flash boiling conditions, including THC, $\mathrm{CO}$, and NOx, generally improved under flash boiling conditions. Such enhancements are regarded as the results of more homogenous fuel-air mixing efficiency under lean-burn conditions.

\section{References}

1. Yang, S., et al., A review on the experimental non-intrusive investigation of fuel injector phase changing flow. Fuel, 2020. 259: p. 116188.

2. Yang, J., et al., Influence of swirl ratio on fuel distribution and cyclic variation under flash boiling conditions in a spark ignition direct injection gasoline engine. Energy Conversion and Management, 2017. 138: $\mathrm{p}$. 565-576.

3. Yang, S., et al., In-nozzle flash boiling flow of multi-component fuel and its effect on near-nozzle spray. Fuel, 2019. 252: p. 55-67.

4. Xiao, D., et al., Evaporation and Condensation of Flash Boiling Sprays Impinging on a Cold Surface Fuel, 2021. 287: p. 119423.

5. Wang, Z., et al., Microscopic and macroscopic characterization of spray impingement under flash boiling conditions with the application of split injection strategy. Fuel, 2018. 212: p. 315-325.

6. $\mathrm{Xu}, \mathrm{Q}$., et al., Investigation of two-hole flash-boiling plume-to-plume interaction and its impact on spray collapse. International Journal of Heat and Mass Transfer, 2019. 138: p. 608-619.

7. Li, Y., et al., Morphology analysis on multi-jet flash-boiling sprays under wide ambient pressures. Fuel, 2018. 211: p. 38-47. 
8. $\quad \mathrm{Du}, \mathrm{J}$., et al., Study of spray collapse phenomenon at flash boiling conditions using simultaneous front and side view imaging. International Journal of Heat and Mass Transfer, 2020. 147: p. 118824.

9. Wu, S., et al., Experimental study of the spray collapse process of multi-hole gasoline fuel injection at flash boiling conditions. Fuel, 2019. 242: p. 109-123.

10. Schulz, F. and F. Beyrau, The influence of flash-boiling on spray-targeting and fuel film formation. Fuel, 2017. 208: p. 587-594

11. Kale, R. and R. Banerjee, Optical investigation of flash boiling and its effect on in-cylinder combustion for butanol isomers and <italic>iso</italic>-octane. International Journal of Engine Research, 2020.

12. Kabasin, D., et al., Heated injectors for ethanol cold starts. SAE International Journal of Fuels and Lubricants, 2009. 2(1): p. 172-179.

13. Huang, Y. and G. Hong, Investigation of the effect of heated ethanol fuel on combustion and emissions of an ethanol direct injection plus gasoline port injection (EDI+GPI) engine. Energy Conversion and Management, 2016. 123: p. 338-347.

14. Fedor, W., et al., GDi Cold Start Emission Reduction with Heated Fuel. 2016, SAE International.

15. Yang, J., et al., Influence of flash boiling spray on the combustion characteristics of a spark-ignition directinjection optical engine under cold start. Combustion and Flame, 2018. 188: p. 66-76.

16. Dong, X., et al., Effects of flash boiling injection on in-cylinder spray, mixing and combustion of a sparkignition direct-injection engine. Proceedings of the Combustion Institute, 2019. 37(4): p. 4921-4928.

17. Nour, M., et al., Effect of flash boiling injection on combustion and PN emissions of DISI optical engine fueled with butanol isomers/TPRF blends. Proceedings of the Combustion Institute, 2021.

18. Li, X., et al., Flash boiling combustion of isomeric butanol and gasoline surrogate blends using constant volume spray chamber and GDI optical engine. Fuel, 2021. 286: p. 119328.

19. Sun, Z., et al., Significant Impact of Flash Boiling Spray on In-Cylinder Soot Formation and Oxidation Process. Energy \& Fuels, 2020. 34(8): p. 10030-10038.

20. Sun, Z., et al., Study of flash boiling combustion with different fuel injection timings in an optical engine using digital image processing diagnostics. Fuel, 2021. 284: p. 119078.

21. Sun, Z., et al., Split Injection Flash Boiling Spray for high efficiency and low emissions in a GDI Engine under Lean Combustion Condition. Proceedings of the Combustion Institute, 2021.

22. Ye, C., et al., Ultra-lean Limit Extension for Gasoline Direct Injection Engine Application via Flash Boiling Atomization. Proceedings of the Combustion Institute, 2021.

23. Ye, C., et al., Effect of discharge current boost on ignition and combustion under cross flow conditions. Combustion and Flame, 2021. 223: p. 1-14.

24. Barone, M., et al. Application of the optical flow method for the experimental analysis of turbulent flame propagation in a transparent engine. in International Conference of Computational Methods in Sciences and Engineering 2016. 2016.

25. Wang, Q., Y. Zhang, and C. Zhao, Experimental investigation of coflow effect on the ignition process of a methane jet diffusion flame. Experimental Thermal and Fluid Science, 2018. 91: p. 184-196. 